Ann. Zootech., I975, 24 (2), 3II-3I3.

NOTE

\title{
INFLUENCE DE LA RESTRICTION \\ DU TEMPS D'ACCÈS A LA BOISSON SUR LA CONSOMMATION ALIMENTAIRE ET LA CROISSANCE DU LAPIN
}

\author{
I. LEBAS et A. DELAVEAL \\ Station do Recherches sur l'Elevage des Porcs, \\ Centre national de Recherches zootechniques, I. N.R. A., \\ r83.50 Jouy cn Josas
}

\section{RÉSUMÉ}

Dix lapins de 7 semaines ont été alimentés ad libitum en eau et en aliment granulé durant 9 jours, puis l'accès ì l'eau ne leur a été permis que $\mathrm{I} / 2$ heure par 24 heures, pour une durée de 8 jours. On obscrve les 2 premiers jours une baisse de la consommation pour l'aliment solide de 53-55 p. Ioo et pour l'aliment liquide de 63-65 p. Ioo. Les consommations augmentent ensuite mais atteignent un maximum de $\mathrm{I}$ Io $\mathrm{g} / \mathrm{j}$ pour l'eau dès le $5^{\mathrm{e}}$ jour. Le temps consacré par les animaux à la prise d'aliments solides suit une évolution semblable.

Les vitesses de croissance moyenne pour la période d'abreuvement libre, les 4 premiers jours d'abreuvement restreint et enfin les 4 derniers, sont respectivement de o $^{\circ} \pm 8,20 \pm 4$, et $t^{6} \pm \mathrm{ro}$ g/jour. Ainsi la restriction de l'alimentation en eau entraîne immédiatement une dépression de consommation qui clure environ 2 jours.

On enregistre généralement des diminutions de consommation et de croissance des lapins dans les élevages de production, à la suite d'abreuvement des animaux insuffisant en quantité ou en fréquencc. Nous avons donc cherché à savoir quelle était la réaction de lapins en croissance à une réduction sensible du temps pendant lequel ils sont à même de s'abreuver.

Dix lapins de 7 semaines, des 2 sexes et de race "Californien "ont été logés clans des cages individuelles munies d'une mangeoire et d'un abreuvoir à surface d'eau libre relié à une éprouvette graduée permettant de contrôler la consommation d'eau. Le poirls moven de départ était de I 327 士 $19 \mathrm{~g}$. Après I 4 jours, dont 9 d'enregistrement, l'accès à l'abreuvoir n'a été permis aux lapins qu'une demi-heure par $2_{4}$ heures (de 9 h 30 à Io h). Cette restriction a été appliquée durant 8 jours. Un système automatique détectant la présence de chaque lapin devant sa mangcoire a permis d'obtenir une indication du temps consacré par les animaux à la consommation d'aliment 
solide. Les quantités d'aliment équilibré granulé (diamètre : $4 \mathrm{~mm}$ ) distribuées et refusées ont été contrôlées chaque jour immédiatement avant la prise de boisson. En fin, le poids des lapins a été contrôlé tous les 4 jours.

Après la réduction du temps d'accès à la boisson, on observe d'abord une baisse très importante de la consommation quotidienne d'aliment solide (-53 p. IOo) et d'eau (-63 p. roo). Ces consommations tendent même à être plus faibles le $2^{\mathrm{e}}$ jour que le premier (fig. $\mathbf{I}$ ). On observe



d'eau de boisson $(0-\mathrm{O})$ et du temps consacré chaque jour aux prises d'aliments solides ( $\square \longrightarrow \square)$

ensuitc une reprise des consommations mais l'ingestion d'eau ne semble pouvoir dépasser i Io $\mathrm{g} /$ jour, tandis que celle d'aliment solide atteint $132 \mathrm{~g}$ le $8 \mathrm{e}$ jour, soit unc ingestion de matière sèche supérieure à celle de l'eau de boisson de près de 20 p. ıoo. Après quelques jours de réduction du temps d'accès à la boisson, les lapins se "précipitent " a fin de boire dès qu'on leur permet d'accéder à l'abrenvoir. La durée de la prise d'eau est égale à io minutes en moyenne; le plus souvent clle est effectuée en une seule fois. Immédiatement après cette ingestion d'eau, on constate toujours une prise d'aliment solide. Au total, le temps consacré à la prise d'aliment solide par 24 heures est réduit de $44 \mathrm{p}$. Ioo le premier jour et d'environ $3^{\circ} \mathrm{p}$. Ioo au delà du $3^{\mathrm{e}}$ jour de restriction de l'accès à l'abreuvoir, soit dans des proportions comparables ì la diminution de l'ingestion d'aliment solide. Ceci peut laisser supposer que la vitesse d'ingestion moyenne serait peu affectée par ce type de restriction en eau. Nous avons par ailleurs, constaté une opposition entre l'importance (durée) de la prise d'aliment solide qui suit celle de la boisson, et les consommations journalières 
d'eau et d'aliment. L'animal qui réalise un repas important sitôt après l'abreuvement semble assoiffé plus rapidement et diminuerait ainsi sa consommation totale journalière.

Enfin, la croissance des animaux est affectée très sensiblement les 4 premiers jours de la restriction (gain moyen quoditien de $20 \pm 2 \mathrm{~g}$ ), mais ne l'est plus les 4 suivants (gain de $46 \pm 5 \mathrm{~g}$ ) puisque pendant la période témoin ( 8 jours) la croissance moyenne quotidienne était de $4^{\circ} \pm 4 \mathrm{~g}$.

Ainsi, la restriction du temps d'accès à l'abreuvoir constitue un choc que les animaux mettent 3 à 4 jours à surmonter, mais en aucun cas la consommation d'eau ne peut alors atteindre des valeurs comparables à celles obtenues avec un abreuvement libre. Au-delà de ces 3-4 jours, nous retrouvons des performances de consommation d'aliment ct de vitesse de croissance proches de celles obtenues dans le cas de l'abreuvement à volonté, comme l'ont montré PRUD'Hon et al. $(1975 b)$ avec une restriction du temps d'accès à l'eau de Io minutes par jour. Enfin, nous avons précisé que la forte réduction de consommation de l'aliment solide se produit les 2 jours suivant l'arrêt de l'abreuvement libre et qu'avec une réduction du temps d'accès à la boisson à $\mathrm{I} / 2$ heure par jour, les lapins peuvent ingérer plus de matière sèche que d'eau contrairement à ce qui est classiquement observé chez le Lapin abreuvé ad libitum (PrUD'Hon et al., 1975 a).

Rę̧u pour publication en décembre 1975.

\section{SUMMARY}

EFFECT OF REDUCED DRINKING TIME ON FEED INTAKE AND GROWTH OF RABBITS

Ten 7 weeks old rabbits received pelleted feed and water ad libitum for 9 days and thereafter, the time allowed for drinking was reduced to $\mathrm{I} / 2 \mathrm{~h}$ per 24 hours during a period of 8 days. The first two days, the solid and liquid food intake decreased : $53-55 \mathrm{p}$. I oo for the pellets and $63-65$ p. Ioo for the water (fig, I). Then the consumption of food and water increased, but in the case of


for the intake of pelleted feed remained in direct relation to the amount eaten.

The mean growth rates recorded during the periods of ad libitum drinking, the first 4 days of restricted drinking and the last 4 days were $40 \pm 8,20 \pm 4$, and $46 \pm$ Io g/day. Thus, water restriction immediately leads to a depression of the feed intake for a period of about 2 days.

\section{RÉFÉRENCES BIBLIOGRAPHIQUES}

Prud'ilon M., Chérubin M., Goussopoulos J., Carles Y., 1975 a. Évolution au cours de la croissance, des caractéristiques de la consommation d'aliments solide et liquide du Lapin domestique nourri ad libitum. Ann. Zootech., 24, 289-298.

Prud'hon M., Chérubin M., Carles Y., Goussopolos J., I975 b. Eiffets de différents niveaux de restriction hydrique sur l'ingestion d'aliments solides par le Lapin. Ann. Zootech., 24, 299-31o. 